\title{
EFFECTS OF FOLIAR SPRAY OF AMINOPLASMAL, NUTRIENTS AND THEIR INTERACTIONS ON VEGETATIVE GROWTH OF PISTACHIO TREES (Pistacia vera L. cv. HALABY)
}

\author{
ZULAIKHA RAMADHAN IBRAHIM and Dr.AZAD AHMAD MAYI \\ Dept. of Horticulture College of Agriculture, University of Duhok, Kurdistan Region-Iraq
}

(Received: November 5, 2018; Accepted for Publication: January 21, 2019)

\begin{abstract}
The present study was conducted in 2016 to evaluate the efficacy of foliar spray of Aminoplasmal (A), Boron (B) and Zinc ( $\mathrm{Zn}$ ) on vegetative growth of pistachio trees (cv. 'Halaby'). Tests were done at a private orchard in the region of Ekmale province of Duhok governorate, Kurdistan Region, Iraq. Tests were designed as a $3 \times 3 \times 3$ factorial experiment in a randomized complete block (RCBD) with three replications. Treatments tested in the study were three concentration levels of Aminoplasmal $\left(0,100\right.$ and $\left.200 \mathrm{ml} . \mathrm{L}^{-1}\right)$; three concentration levels of Boron $\left(0,200\right.$, and $\left.300 \mathrm{mg}^{-L^{-1}}\right)$ and three concentration levels of Zinc $(0,400$, and $\left.600 \mathrm{mg} . \mathrm{L}^{-1}\right)$. Treatments were consisted of 81 trees with three replicates and each replicate was contained 27 trees, foliar spraying of studied elements applied at two time, one in time of swollen bud and the other repeated after one month (23March and 27April 2016). Different trait, such as shoot lengths and diameters, leaf area, leaf dry weight, leaf chlorophyll content and number of leave per shoot were measured. Based on the results, the effects of spraying elements improved the vegetative characteristic. Results showed that Aminoplasmal at second and third levels had a significant effect on shoot lengths and diameters, leaf area, leaf dry weight as compared to control, on the other hand there were no significant differences between the level of Aminoplasmal $100 \mathrm{ml} . \mathrm{L}^{-1}$ and $200 \mathrm{ml} . \mathrm{L}^{-1}$ on shoot lengths and diameters, leaf area, leaf dry weight. In contrasts both of Boron at level 300mg. $\mathrm{L}^{-1}$ and Zinc at level $600 \mathrm{mg} . \mathrm{L}^{-1}$ had a significant effect on total leaf chlorophyll content and number of leave per shoot. Referring to the triple interaction, the highest shoot length was obtained in the trees receiving $100 \mathrm{ml} . \mathrm{L}-1$ Aminoplasmal $\times 300 \mathrm{mg} . \mathrm{L}-1$ Boron $\times 600 \mathrm{mg} . \mathrm{L}-1$ Zinc. Whereas the highest shoot diameter, Single leaf area, leaf dry weight, Total chlorophyll content, Number of leaves per shoot was obtained from the tree received Aminoplasmal200 ml. $\mathrm{L}^{-1}$ with Boron $300 \mathrm{mg} . \mathrm{L}^{-1}$ and 600 mg.L ${ }^{-1}$ Zinc.
\end{abstract}

KEYWORD: Pistachio, Amino plasmal, Boron and Zinc

\section{INTRODUCTION}

$\mathbf{P}$ istachio (Pistacia vera L.) a deciduous, and wind-pollinated tree species, is the member of the family anacardiaceae, there are about eleven species of pistachio trees but $P$. vera is the only species grown commercially because it produces fruit of suitable size to be marketed. Species such as $P$. atlantica, $P$. terebinthus and $P$. integerrima are used as rootstocks for $P$. vera. The pistachio's origin is still uncertain, but most competent agree that it may be originated in Asia Minor. Iran and United States are the first and second and then Turkey, Syria are most pistachio producer countries in the world, respectively (FAO, 2015).
Aminoplasmal are behold as precursor and constituents of the proteins (Rai, 2002), which are important for stimulation of cell growth. They include both acid and basic groups and work as a buffers, which aid to maintain favorable or indirectly influence the physiological activities in plant growth and development such as exogenous application of amino acids have been reported to modulate the growth (Shiraishi et al., 2010). However, effects of foliar application of aminoplasmal on the growth, functionally, amino acids mostly L- amino acids rather than D- amino acids are involved in the enzymes reliable for the structural photosynthesis process. Furthermore aminoplasmal act as chelating effect on micronutrients. When used together with micronutrients. The absorption and transportation 
of micronutrients inside the plant is easier (Abo El-Magd et al., 2015). The application of amino acids as foliar spray is based on their requirement by plant in general and critical stages of growth in particular (Coruzzi and Last 2000). Furthermore, amino acids can also be an important source of available nitrogen for plants (Rahdari and Panahi, 2012). Yet amino acids are fundamental in chlorophyll production; chlorophyll being the driving force behind photosynthesis. Amino acids help to increase chlorophyll concentration in the plant, leading to higher degree of photosynthesis, which in turn leads to even more available energy (Molaie et al., 2013).

Boron acts an important role in pollen germination and pollen tube growth (Storey, 2007). Boron is a major element for the growth and development of healthy plant. But the method in which B applied is also important so the amount of boron needed for normal growth of pistachio leaves is different from the amount of boron needed for the growth of reproductive buds, flower production and pollination.(Brown et al., 1995; Wojcik et al., 2003; Ganie et al., 2013). Furthermore results showed that by adding boron to pistachio tree lead to enhance fruit quantity and quality (Seyyedi, 1998).

Zinc is a cofactor of over 300 enzymes, proteins and has an early and specific influence on cell division, nucleic acid metabolism, and protein synthesis (Marschner, 1986). Zinc deficiency in walnut is visually expressed as small leaves and nuts, delayed opening of vegetative and flower buds, leaf chlorosis between the lateral veins, wavy leaves with upward folded leaf margins and terminal dieback (Ramos, 1997). Foliar application of $\mathrm{Zn}$ has been successfully applied to promote tree vigor (Wojcik, 2007). Both B and Zn applications have been observed to have a positive effect on chlorophyll contents in B and $\mathrm{Zn}$ deficient plants (Zheng et al., 1989). Boron and Zinc deficiencies are more probable early in the season because the moving of elements from the root to the aboveground portion may not be adequate before leaf expansion (Neilsen et al., 2004). The effects of Aminoplasmal, Boron, Zinc and their interactions on vegetative growth of pistachio trees (cv. 'Halaby') were evaluated in this experiment. Therefore, the aim of the present study was to improve pistachio vegetative growth by using Aminoplasmal, Boron and Zinc spraying.

\section{MATRIALS AND METHODS \\ Plant material and experiments}

This study was conducted on pistachio (cv. 'Halaby') trees were 14 years old, at a private orchard at Ekmale province of Duhok governorate, Kurdistan Region, in 2016.The orchard was rainfed (nonirrigated) orchard, to investigate the effect of Amino plasmal, Boron, Zinc, and their interactions on the vegetative growth of Pistachio tree, the foliar spraying of studied elements was done during swelling of the bud and repeated after one month (23March and 27April 2016). Amino acid was supplied from Aminoplasmal B. Braun $10 \%$ (B. Braun Melsungen AG), in three levels $(0,100$ and 200 ml.L-1). The commercial product 'Amino plasmal" was used as a source of amino acid. In the amino plasma, total amino acid $100 \mathrm{~g} / \mathrm{L}$; total $\mathrm{N}(15.8 \mathrm{~g} / \mathrm{L})$ and 17 different amino acids are present viz., Isoleucine $(5.00 \mathrm{~g})$, Leucine $(8.90 \mathrm{~g})$, Lysinehydrochloride $(8.56 \mathrm{~g})$, Methionine $(4.40 \mathrm{~g})$, Phenylalanine (4.70g), Threonine (4.20g), Tryptophane $(1.60 \mathrm{~g})$, Valine, $(6.20 \mathrm{~g})$, Arginine $(11.50 \mathrm{~g})$, Histidine $(3.00 \mathrm{~g})$, Glycine $(12.00 \mathrm{~g})$, Alanine (10.50g), Proline (5.50g), Aspartic acid (5.60g), Glutamic acid (7.20g), Tyrosine $(0.40 \mathrm{~g})$, Serine $(2.30 \mathrm{~g})$. Boron was supplied from Boric acid in three levels $\left(0,200\right.$, and $\left.300 \mathrm{mg} . \mathrm{L}^{-1}\right)$ and Zinc was supplied from Zinc sulfate source in three levels $\left(0,400\right.$, and $\left.600 \mathrm{mg} . \mathrm{L}^{-1}\right)$. Treatments were consisted of 81 trees with three replicates; with (1) tree for each experimental unit, treatment were distributed in factorial arrangement use's Randomized Complete Block Design(RCBD) (Al-Rawi and Khalaf-Alla 2000).

The lengths and diameters of current year branches measured in late winter (February) by electric vernier. The number of leaves per shoot, single leaf area, leaf dry weight and leaf chlorophyll content of current year branches measured at the end of first growth(in late august). Leaf area was measured by a Digital Leaf Area Meter (ADC, Bio scientific LTD), 10 leaves (at middle of shoot) were used for measuring leaf area in each replication. Leaf dry weight after the leaves fresh weight was taken; they were oven dried at $70^{\circ} \mathrm{C}$ until weight fixing (Gobara, 1998). Weight was taken by electrical balance $0.00 \mathrm{~g}$. Leaf chlorophyll content of current year branches measured by using Chlorophyll Meter, SPAD502 , Konica Minolta. and number of leaves per shoot was counted. 


\section{Statistical analysis}

The data were analyzed statistically by using SAS system (SAS, 2001). The significant differences among means were carried out by using Duncan multiple at 0.05 level.

\section{RESULTS}

Data as shown in table (1): Clear that aminoplasmal, at second and third levels significantly increased shoot length, compared with the control, while boron and zinc significantly increased shoot length at third level $(42.84,43.61 \mathrm{~cm})$ at concentration $300 \mathrm{mg} . \mathrm{L}^{-1}$ and $600 \mathrm{mg} . \mathrm{L}^{-1}$ respectively. Also the highest shoot length $(46.70 \mathrm{~cm})$ was at concentrations of $\left(100 \mathrm{ml} . \mathrm{L}^{-1}\right.$ aminoplasmal $\times 300 \mathrm{mg} . \mathrm{L}^{-1}$ boron $\times$ $600 \mathrm{mg} . \mathrm{L}^{-1}$ zinc). Whereas, the lowest shoot length $(23.99 \mathrm{~cm})$ noticed in the untreated treatments.

Table(1): Effects of foliar spray of Aminoplasmal(A), Boron(B), Zinc(Zn) and their interactions on shoot length $(\mathrm{cm})$ of pistachio tree $\mathrm{cv}$. 'Halaby'.

\begin{tabular}{|c|c|c|c|c|c|c|}
\hline \multirow{2}{*}{$\begin{array}{c}\text { Amino } \\
\text { plasmal } \mathbf{m l . L ^ { - 1 }}\end{array}$} & \multirow[t]{2}{*}{ Boron mg.L.$^{-1}$} & \multicolumn{3}{|c|}{ Zinc $\mathbf{m g} . \mathbf{L}^{-1}$} & \multirow[t]{2}{*}{$\mathrm{A} \times \mathrm{B}$} & \multirow[t]{2}{*}{ Means of A } \\
\hline & & 0 & 400 & 600 & & \\
\hline \multirow[t]{3}{*}{0} & 0.0 & $23.99 \mathrm{i}$ & $32.86 \mathrm{gh}$ & $34.23 \mathrm{f}-\mathrm{h}$ & $30.36 \mathrm{~d}$ & $34.97 b$ \\
\hline & 200 & $25.57 \mathrm{i}$ & $36.97 \mathrm{~d}-\mathrm{g}$ & $43.40 \mathrm{a}-\mathrm{d}$ & $35.31 \mathrm{c}$ & \\
\hline & 300 & $29.20 \mathrm{hi}$ & $43.03 \mathrm{a}-\mathrm{d}$ & $45.47 \mathrm{ab}$ & $39.23 \mathrm{~b}$ & \\
\hline \multirow[t]{3}{*}{100} & 0 & $35.47 \mathrm{e}-\mathrm{h}$ & $40.71 \mathrm{a}-\mathrm{f}$ & $43.33 a-d$ & $39.84 b$ & $41.98 \mathrm{a}$ \\
\hline & 200 & $37.80 \mathrm{c}-\mathrm{g}$ & $42.40 \mathrm{a}-\mathrm{e}$ & $44.80 \mathrm{a}-\mathrm{c}$ & 41.67ab & \\
\hline & 300 & $41.77 \mathrm{a}-\mathrm{e}$ & $44.80 \mathrm{a}-\mathrm{c}$ & $46.70 \mathrm{a}$ & $44.42 \mathrm{a}$ & \\
\hline \multirow[t]{3}{*}{200} & 0 & $38.13 b-g$ & $41.48 \mathrm{a}-\mathrm{e}$ & $43.69 a-d$ & 41.10ab & $42.82 \mathrm{a}$ \\
\hline & 200 & $41.10 \mathrm{a}-\mathrm{f}$ & $42.13 \mathrm{a}-\mathrm{e}$ & $44.32 \mathrm{a}-\mathrm{c}$ & $42.52 \mathrm{ab}$ & \\
\hline & 300 & $43.39 \mathrm{a}-\mathrm{d}$ & $44.63 a-c$ & $46.53 \mathrm{a}$ & $44.85 \mathrm{a}$ & \\
\hline \multicolumn{2}{|c|}{ Means of Zinc } & $35.16 \mathrm{c}$ & $41.00 \mathrm{~b}$ & $43.61 \mathrm{a}$ & \multirow{4}{*}{\multicolumn{2}{|c|}{ Means of Boron }} \\
\hline \multirow[t]{3}{*}{$\mathbf{A} \times \mathbf{Z n}$} & 0 & $26.25 \mathrm{e}$ & $37.62 \mathrm{~d}$ & $41.03 \mathrm{~b}-\mathrm{d}$ & & \\
\hline & 100 & $38.34 d$ & $42.64 a-c$ & $44.94 \mathrm{a}$ & & \\
\hline & 200 & $40.87 \mathrm{~cd}$ & $42.75 \mathrm{a}-\mathrm{c}$ & $44.85 \mathrm{ab}$ & & \\
\hline \multirow[t]{3}{*}{$\mathbf{B} \times \mathbf{Z n}$} & 0 & $32.53 \mathrm{e}$ & $38.35 \mathrm{~cd}$ & $40.42 \mathrm{bc}$ & \multicolumn{2}{|c|}{$37.10 \mathrm{c}$} \\
\hline & 200 & $34.82 \mathrm{de}$ & $40.50 \mathrm{bc}$ & 44.17ab & \multicolumn{2}{|c|}{$39.83 b$} \\
\hline & 300 & $38.12 \mathrm{~cd}$ & $44.16 \mathrm{ab}$ & $46.23 \mathrm{a}$ & \multicolumn{2}{|c|}{$42.84 \mathrm{a}$} \\
\hline
\end{tabular}

Mean with a column, row and their interaction following with the same latter are not significantly different according to Duncan multiple at 0.05 level.

The result in table (2): reveal that aminoplasmal significantly affected on shoot diameter level at concentration, $200 \mathrm{ml} . \mathrm{L}^{-1}$ and $300 \mathrm{ml} . \mathrm{L}^{-1}$. Whereas, diameter increased by increment of boron up to $300 \mathrm{mg} . \mathrm{L}^{-1}$ and zinc up to $600 \mathrm{mg} . \mathrm{L}^{-1},(9.67,9.63,9.90 \mathrm{~mm})$ respectively.
Referring to the triple interactions, the highest shoot diameter $(11.54 \mathrm{~mm})$ was obtained from the interactions of aminoplasmal $\left(200 \mathrm{ml} . \mathrm{L}^{-1}\right)$ plus boron $\left(300 \mathrm{mg} . \mathrm{L}^{-1}\right)$ and zinc $\left(600 \mathrm{mg} . \mathrm{L}^{-1}\right)$, while the lowest shoot diameter was obtained from the control
$(6.71 \mathrm{~mm})$.

Table (2): Effect of foliar spray of Aminoplasmal(A), Boron(B), Zinc(Zn) and their interactions on shoot diameter $(\mathrm{mm})$ of pistachio tree cv. 'Halaby'.

\begin{tabular}{|c|c|c|c|c|c|c|}
\hline \multirow{2}{*}{$\begin{array}{c}\text { Amino } \\
\text { plasmal } \mathrm{ml}^{-\mathrm{L}^{-1}}\end{array}$} & \multirow{2}{*}{$\begin{array}{l}\text { Boron } \\
\mathrm{mg}^{-\mathrm{L}^{-1}}\end{array}$} & \multicolumn{3}{|c|}{ Zinc $\mathrm{mg}^{-\mathrm{L}^{-1}}$} & \multirow[t]{2}{*}{$A \times B$} & \multirow[t]{2}{*}{$A$} \\
\hline & & 0 & 400 & 600 & & \\
\hline \multirow[t]{2}{*}{0} & 0.0 & $6.71 \mathrm{i}$ & 7.55hi & $7.92 \mathrm{~g}-\mathrm{i}$ & $7.39 f$ & $8.09 b$ \\
\hline & 200 & 7.63hi & $8.20 \mathrm{~g}-\mathrm{i}$ & $8.82 \mathrm{~d}-\mathrm{h}$ & $8.22 e$ & \\
\hline
\end{tabular}




\begin{tabular}{|c|c|c|c|c|c|c|}
\hline & 300 & $8.16 \mathrm{~g}-\mathrm{i}$ & $8.81 \mathrm{~d}-\mathrm{h}$ & $9.04 c-h$ & 8.67de & \\
\hline \multirow[t]{3}{*}{100} & 0 & $8.26 \mathrm{~g}-\mathrm{i}$ & $9.00 \mathrm{~d}-\mathrm{h}$ & $9.33 c-g$ & $8.86 \mathrm{c}-\mathrm{e}$ & $9.46 a$ \\
\hline & 200 & $8.52 f-h$ & $9.60 \mathrm{~b}-\mathrm{g}$ & $10.32 a-e$ & 9.48a-d & \\
\hline & 300 & 9.03d-h & $10.03 a-f$ & $11.06 a b$ & $10.04 a b$ & \\
\hline \multirow[t]{3}{*}{200} & 0 & $8.26 \mathrm{~g}-\mathrm{i}$ & $9.06 c-h$ & $10.35 a-d$ & $9.23 b-d$ & $9.67 a$ \\
\hline & 200 & $8.64 \mathrm{e}-\mathrm{h}$ & $9.47 \mathrm{~b}-\mathrm{g}$ & $10.69 a-c$ & $9.60 a-c$ & \\
\hline & 300 & $9.04 c-h$ & $9.97 a-f$ & $11.54 a$ & $10.18 a$ & \\
\hline \multicolumn{2}{|c|}{ Zn } & $8.25 c$ & $9.08 b$ & $9.90 \mathrm{a}$ & \multirow{4}{*}{\multicolumn{2}{|c|}{ Means of Boron }} \\
\hline \multirow[t]{3}{*}{$A \times Z n$} & 0 & $7.50 \mathrm{~d}$ & $8.18 \mathrm{~cd}$ & $8.59 c$ & & \\
\hline & 100 & $8.60 c$ & $9.54 b$ & 10.24ab & & \\
\hline & 200 & $8.65 c$ & $9.50 \mathrm{~b}$ & $10.86 a$ & & \\
\hline \multirow[t]{3}{*}{$B \times Z n$} & 0 & $7.74 f$ & $8.54 d-f$ & $9.20 b-d$ & \multicolumn{2}{|c|}{$8.49 c$} \\
\hline & 200 & $8.26 \mathrm{ef}$ & $9.09 b-e$ & $9.95 a b$ & \multicolumn{2}{|c|}{$9.10 \mathrm{~b}$} \\
\hline & 300 & $8.74 c-\mathrm{e}$ & $9.60 b-d$ & $10.55 a$ & \multicolumn{2}{|c|}{$9.63 a$} \\
\hline
\end{tabular}

Mean with a column, row and their interaction following with the same latter are not significantly different according to Duncan multiple at 0.05 level.

Data in table (3): reveals that aminoplasmal in both concentration significantly affected on leaf area as compared with the control, whereas, leaf area increased by increment of boron to $300 \mathrm{mg} . \mathrm{L}^{-1}$ and zinc to $600 \mathrm{mg} . \mathrm{L}^{-1},\left(137.73 \mathrm{~cm}^{2}, 137.12 \mathrm{~cm}^{2}\right.$, $135.46 \mathrm{~cm}^{2}$ ) respectively. The interactions of aminoplasmal, boron and zinc indicate significant differences in leaf area. The highest leaf area $\left(145.97 \mathrm{~cm}^{2}\right)$ was obtained when the trees treated with aminoplasmal $\left(200 \mathrm{ml} . \mathrm{L}^{-1}\right)$, boron (300mg.L $\left.{ }^{1}\right)$ and zinc $\left(600 \mathrm{mg} . \mathrm{L}^{-1}\right)$. In contrast, the lowest leaf area $\left(88.521 \mathrm{~cm}^{2}\right)$ was recorded in the untreated treatment.

Table (3): Effect of foliar spray of Aminoplasmal(A), Boron(B), Zinc(Zn) and their interactions on single leaf area $\left(\mathrm{cm}^{2}\right)$ of pistachio tree cv. 'Halaby'.

\begin{tabular}{|c|c|c|c|c|c|c|}
\hline \multirow{2}{*}{$\begin{array}{c}\text { Amino } \\
\text { plasmal } \mathrm{ml}^{-\mathrm{L}^{-1}}\end{array}$} & \multirow{2}{*}{$\begin{array}{l}\text { Boron } \\
\mathrm{mg} \cdot \mathrm{L}^{-1}\end{array}$} & \multicolumn{3}{|c|}{ Zinc $\mathrm{mg}^{-\mathrm{L}^{-1}}$} & \multirow[t]{2}{*}{$A \times B$} & \multirow[t]{2}{*}{ Means of $A$} \\
\hline & & 0 & 400 & 600 & & \\
\hline \multirow[t]{3}{*}{0} & 0.0 & $88.52 \mid$ & $119.12 \mathrm{ij}$ & $124.03 \mathrm{~h}-\mathrm{j}$ & $110.56 f$ & $118.69 b$ \\
\hline & 200 & $110.89 k$ & $123.60 \mathrm{~h}-\mathrm{j}$ & 126.32hi & $120.27 e$ & \\
\hline & 300 & $121.33 \mathrm{ij}$ & $125.72 h-j$ & $128.68 f-h$ & $125.24 d$ & \\
\hline \multirow[t]{3}{*}{100} & 0 & $124.14 \mathrm{~h}-\mathrm{j}$ & $125.14 \mathrm{~h}-\mathrm{j}$ & $134.48 \mathrm{ef}$ & $127.92 \mathrm{~cd}$ & $135.70 \mathrm{a}$ \\
\hline & 200 & $133.71 \mathrm{e}-\mathrm{g}$ & 139.39a-e & $140.51 \mathrm{a}-\mathrm{e}$ & $137.87 \mathrm{~b}$ & \\
\hline & 300 & $140.27 a-e$ & $141.69 a-c$ & $142.02 a-c$ & $141.32 \mathrm{ab}$ & \\
\hline \multirow[t]{3}{*}{200} & 0 & 126.79hi & $127.84 \mathrm{~g}-\mathrm{i}$ & $135.80 c-e$ & $130.14 \mathrm{c}$ & 137.73a \\
\hline & 200 & $135.25 c-f$ & $138.15 b-e$ & 141.38a-d & $138.26 b$ & \\
\hline & 300 & $143.51 \mathrm{ab}$ & $144.91 \mathrm{ab}$ & $145.97 a$ & $144.80 a$ & \\
\hline \multicolumn{2}{|c|}{ Means of Zinc } & $124.93 c$ & $131.73 b$ & $135.46 a$ & \multirow{4}{*}{\multicolumn{2}{|c|}{ Means of B }} \\
\hline \multirow[t]{3}{*}{$A \times Z n$} & 0 & $106.91 \mathrm{f}$ & $122.81 \mathrm{e}$ & $126.34 d$ & & \\
\hline & 100 & $132.71 \mathrm{c}$ & $135.41 b c$ & $139.00 \mathrm{ab}$ & & \\
\hline & 200 & $135.19 b c$ & $136.96 \mathrm{~b}$ & $141.05 a$ & & \\
\hline \multirow[t]{3}{*}{$B \times Z n$} & 0 & $113.15 \mathrm{e}$ & $124.03 d$ & $131.43 c$ & \multicolumn{2}{|c|}{$122.87 \mathrm{c}$} \\
\hline & 200 & $126.62 d$ & $133.71 b c$ & 136.07ab & \multicolumn{2}{|c|}{$132.13 b$} \\
\hline & 300 & $135.04 \mathrm{bc}$ & 137.44ab & $138.89 a$ & \multicolumn{2}{|c|}{ 137.12a } \\
\hline
\end{tabular}

Mean with a column, row and their interaction following with the same latter are not significantly different according to Duncan multiple at 0.05 level. 
It is lucidity recognized in table (4): That leaf dry weight increased significantly when aminoplasmal level increased as compared with the control, on the other hand there were no significant differences between the level of aminoplasmal $100 \mathrm{ml} . \mathrm{L}^{-1}$ and $200 \mathrm{ml} . \mathrm{L}^{-1}$. While, boron at (300mg. $\left.\mathrm{L}^{-1}\right)$ and zinc $\left(600 \mathrm{mg} \cdot \mathrm{L}^{-1}\right)$ increased leaf dry weight significantly $(4.28 \mathrm{~g})$ and (4.51g) respectively. Concerning the interactions of aminoplasmal $\times$ boron $\times$ zinc gave the highest value of leaf dry weight $(4.96 \mathrm{~g})$ at levels $200 \mathrm{ml} . \mathrm{L}^{-}$ 1 aminoplasmal $\times 300 \mathrm{mg} . \mathrm{L}^{-1}$ boron $\times 600 \mathrm{mg} . \mathrm{L}^{-1}$ zinc. Whereas the lowest leaves dry weight $(2.45 \mathrm{~g})$ was recorded in untreated treatment.

Table (4): Effect of foliar spray of Aminoplasmal(A), Boron(B), Zinc(Zn) and their interactions on leaf dry weight $(\mathrm{g})$ of pistachio tree cv. 'Halaby'.

\begin{tabular}{|c|c|c|c|c|c|c|}
\hline \multirow{2}{*}{$\begin{array}{c}\text { Amino } \\
\text { plasmal } \mathrm{ml}^{-\mathrm{L}^{-1}}\end{array}$} & \multirow[t]{2}{*}{ Boron $\mathrm{mg} \cdot \mathrm{L}^{-1}$} & \multicolumn{3}{|c|}{ Zinc $\mathbf{m g} \mathrm{L}^{-1}$} & \multirow[t]{2}{*}{$A \times B$} & \multirow[t]{2}{*}{ Means of $A$} \\
\hline & & 0 & 400 & 600 & & \\
\hline \multirow[t]{3}{*}{0} & 0.0 & $2.45 \mathrm{~m}$ & 3.181 & $4.17 \mathrm{fg}$ & $3.27 d$ & $3.71 b$ \\
\hline & 200 & 3.33j-I & $3.57 \mathrm{~h}-\mathrm{I}$ & $4.32 \mathrm{c}-\mathrm{e}$ & $3.74 \mathrm{c}$ & \\
\hline & 300 & $3.80 f-i$ & $3.78 \mathrm{~g}-\mathrm{i}$ & $4.82 \mathrm{ab}$ & $4.13 b$ & \\
\hline \multirow[t]{3}{*}{100} & 0 & 3.161 & $3.72 \mathrm{~h}-\mathrm{j}$ & $4.20 \mathrm{ef}$ & $3.69 c$ & $3.95 a$ \\
\hline & 200 & $3.20 \mathrm{kl}$ & $3.84 \mathrm{f}-\mathrm{h}$ & $4.57 a-e$ & $3.87 \mathrm{c}$ & \\
\hline & 300 & $3.52 \mathrm{~h}-\mathrm{I}$ & $4.63 a-d$ & $4.74 a-c$ & $4.30 \mathrm{ab}$ & \\
\hline \multirow[t]{3}{*}{200} & 0 & 3.171 & $3.62 \mathrm{~h}-\mathrm{k}$ & $4.27 \mathrm{de}$ & $3.69 c$ & $4.07 a$ \\
\hline & 200 & $3.38 \mathrm{i}-1$ & $4.43 \mathrm{~b}-\mathrm{e}$ & $4.55 \mathrm{a}-\mathrm{e}$ & $4.12 \mathrm{~b}$ & \\
\hline & 300 & $3.42 \mathrm{~h}-\mathrm{I}$ & $4.82 \mathrm{ab}$ & $4.96 \mathrm{a}$ & $4.40 \mathrm{a}$ & \\
\hline \multicolumn{2}{|c|}{ Means of Zinc } & $3.27 \mathrm{c}$ & $3.95 b$ & $4.51 \mathrm{a}$ & \multirow{4}{*}{\multicolumn{2}{|c|}{ Means of $B$}} \\
\hline \multirow[t]{3}{*}{$A \times Z n$} & 0 & $3.19 \mathrm{e}$ & $3.51 \mathrm{~d}$ & 4.44ab & & \\
\hline & 100 & 3.29de & $4.06 \mathrm{c}$ & $4.50 a b$ & & \\
\hline & 200 & 3.32de & $4.29 \mathrm{~b}$ & $4.59 \mathrm{a}$ & & \\
\hline \multirow[t]{3}{*}{$B \times Z n$} & 0 & $2.93 \mathrm{~g}$ & 3.51 ef & $4.22 \mathrm{c}$ & \multicolumn{2}{|c|}{$3.55 c$} \\
\hline & 200 & $3.31 \mathrm{f}$ & $3.94 d$ & $4.48 b$ & \multicolumn{2}{|c|}{$3.91 \mathrm{~b}$} \\
\hline & 300 & $3.58 \mathrm{e}$ & $4.41 \mathrm{bc}$ & $4.84 a$ & \multicolumn{2}{|c|}{$4.28 \mathrm{a}$} \\
\hline
\end{tabular}

Mean with a column, row and their interaction following with the same latter are not significantly different according to Duncan multiple at 0.05 level.

Table (5): Indicates that aminoplasmal application significantly increased total chlorophyll content in leaf at concentrations $200 \mathrm{ml} . \mathrm{L}^{-1}$ and $300 \mathrm{ml} . \mathrm{L}^{-1}$, on the other hand boron and zinc application had a significant effect on total chlorophyll content so increasing the level of boron up to $300 \mathrm{mg} . \mathrm{L}^{-1}$ and zinc up to $600 \mathrm{mg} . \mathrm{L}^{-1}$ lead to significant increase in total chlorophyll content, Whereas, leaf total chlorophyll content increased at combinations between $200 \mathrm{ml} . \mathrm{L}^{-1}$ aminoplasmal plus boron $300 \mathrm{mg} . \mathrm{L}^{-1}$ and zinc $600 \mathrm{mg} . \mathrm{L}^{-1}$ was $(54.11 \%)$ respectively. in contrast total chlorophyll content in untreated trees gave the lowest value $(41.20 \%)$

Table (5): Effect of foliar spray of Aminoplasmal(A), Boron(B), Zinc(Zn) and their interactions on leaf chlorophyll of pistachio tree cv. 'Halaby'.

\begin{tabular}{|c|c|c|c|c|c|c|}
\hline \multirow{2}{*}{$\begin{array}{c}\text { Amino } \\
\text { plasmal } \mathrm{ml}^{-\mathrm{L}^{-1}}\end{array}$} & \multirow[t]{2}{*}{ Boron $\mathrm{mg} \cdot \mathrm{L}^{-1}$} & \multicolumn{3}{|c|}{ Zinc $\mathrm{mg} \mathrm{L}^{-1}$} & \multirow[t]{2}{*}{$A \times B$} & \multirow[t]{2}{*}{ Means of $A$} \\
\hline & & 0 & 400 & 600 & & \\
\hline \multirow[t]{3}{*}{0} & 0.0 & $41.20 p$ & $45.83 \mathrm{~nm}$ & $47.59 \mathrm{j}-\mathrm{m}$ & $44.88 \mathrm{e}$ & 46.30 \\
\hline & 200 & 43.080 & $46.16 \mathrm{mn}$ & $48.86 \mathrm{~g}-\mathrm{j}$ & $46.03 d$ & C \\
\hline & 300 & $46.54 i-n$ & $47.17 \mathrm{k}-\mathrm{n}$ & $50.27 \mathrm{~d}-\mathrm{g}$ & $47.99 \mathrm{c}$ & \\
\hline 100 & 0 & $45.73 n$ & $47.23 \mathrm{k}-\mathrm{n}$ & $49.31 \mathrm{~g}-\mathrm{j}$ & $47.43 \mathrm{c}$ & 49.69 \\
\hline
\end{tabular}




\begin{tabular}{|c|c|c|c|c|c|c|}
\hline & 200 & $48.38 \mathrm{i}-\mathrm{k}$ & $50.08 \mathrm{~d}-\mathrm{h}$ & $52.12 \mathrm{bc}$ & $50.19 b$ & B \\
\hline & 300 & $49.61 d-i$ & $51.31 \mathrm{~cd}$ & $53.43 a b$ & $51.45 a$ & \\
\hline \multirow[t]{3}{*}{200} & 0 & $47.99 i-1$ & $50.75 c-f$ & $51.18 c-e$ & $49.97 \mathrm{~b}$ & 50.83 \\
\hline & 200 & $48.48 \mathrm{~g}-\mathrm{k}$ & $50.88 c-f$ & $52.19 \mathrm{bc}$ & $50.52 b$ & A \\
\hline & 300 & $49.38 \mathrm{~g}-\mathrm{j}$ & $52.48 a-c$ & $54.11 a$ & $51.99 a$ & \\
\hline \multicolumn{2}{|c|}{ Means of Zinc } & $46.71 \mathrm{c}$ & $49.10 \mathrm{~b}$ & $51.00 a$ & \multirow{4}{*}{\multicolumn{2}{|c|}{ Means of Boron }} \\
\hline \multirow[t]{3}{*}{$A \times Z n$} & 0 & $43.61 f$ & $46.39 \mathrm{e}$ & $48.91 \mathrm{c}$ & & \\
\hline & 100 & $47.91 d$ & $49.54 c$ & $51.62 a b$ & & \\
\hline & 200 & $48.61 \mathrm{~cd}$ & $51.37 \mathrm{~b}$ & $52.49 a$ & & \\
\hline \multirow[t]{3}{*}{$B \times Z n$} & 0 & $44.98 f$ & $47.94 d$ & $49.36 c$ & \multicolumn{2}{|c|}{$47.42 \mathrm{c}$} \\
\hline & 200 & $46.65 \mathrm{e}$ & $49.04 c$ & $51.06 \mathrm{~b}$ & \multicolumn{2}{|c|}{$48.91 b$} \\
\hline & 300 & $48.51 \mathrm{~cd}$ & $50.32 b$ & $52.60 a$ & \multicolumn{2}{|c|}{$50.48 a$} \\
\hline
\end{tabular}

Mean with a column, row and their interaction following with the same latter are not significantly different according to Duncan multiple at 0.05 level.

Table (6): Results show that number of leave. Shoot were increased with the increasing the levels of aminoplasmal up to $200 \mathrm{ml}^{-\mathrm{L}^{-1}}$ boron up to $300 \mathrm{mg} . \mathrm{L}^{-1}$ and zinc up to $600 \mathrm{mg} . \mathrm{L}^{-1}$, $(20.37$, 20.04 and 20.38) respectively. On the other hand there are a significant difference between the level of aminoplasmal $100 \mathrm{ml} \cdot \mathrm{L}^{-1}$ and $200 \mathrm{ml} . \mathrm{L}^{-1}$, the results indicate that spray application of boron at second level had a significant difference with the third level application. The number of leave.shoot sharply increased at application third level of zinc and had a significant difference with the second level of zinc application. Data in table (6) also showed that the combinations between aminoplasmal $\times$ boron $\times$ zinc at levels $200 \mathrm{ml} . \mathrm{L}^{-1}$ $\times 300 \mathrm{mg} . \mathrm{L}^{-1} \times 600 \mathrm{mg} . \mathrm{L}^{-1}$ gave the highest number of leave.shoot (25.25). Whereas, the lowest number of leave.shoot (11.83) was recorded in control

treatments.

Table (6): Effect of foliar spray of Aminoplasmal(A), Boron(B), Zinc(Zn) and their interactions on number of leaves.shoot of pistachio tree cv. 'Halaby'.

\begin{tabular}{|c|c|c|c|c|c|c|}
\hline \multirow{2}{*}{$\begin{array}{c}\text { Amino } \\
\text { plasmal ml. } \mathrm{L}^{-1}\end{array}$} & \multirow[t]{2}{*}{ Boron $\mathrm{mg} \cdot \mathrm{L}^{-1}$} & \multicolumn{3}{|c|}{ Zinc $\mathbf{m g} \cdot \mathrm{L}^{-1}$} & \multirow[t]{2}{*}{$A \times B$} & \multirow[t]{2}{*}{ A } \\
\hline & & 0 & 400 & 600 & & \\
\hline \multirow[t]{3}{*}{0} & 0.0 & $11.83 \mathrm{j}$ & 13.33ij & $16.92 \mathrm{e}-\mathrm{i}$ & $14.03 f$ & $16.29 c$ \\
\hline & 200 & $14.33 \mathrm{~h}-\mathrm{j}$ & $17.25 \mathrm{e}-\mathrm{h}$ & $18.47 \mathrm{c}-\mathrm{g}$ & 16.68de & \\
\hline & 300 & $16.17 f-i$ & $17.90 \mathrm{~d}-\mathrm{h}$ & $20.42 \mathrm{~b}-\mathrm{e}$ & $18.16 c-d$ & \\
\hline \multirow[t]{3}{*}{100} & 0 & $13.36 \mathrm{ij}$ & $15.25 \mathrm{~g}-\mathrm{j}$ & $19.53 c-f$ & $16.05 \mathrm{e}$ & $17.56 \mathrm{~b}$ \\
\hline & 200 & $14.32 \mathrm{~h}-\mathrm{j}$ & $17.48 \mathrm{e}-\mathrm{h}$ & 20.01b-f & $17.27 \mathrm{de}$ & \\
\hline & 300 & $17.50 \mathrm{e}-\mathrm{h}$ & $19.17 c-f$ & $21.42 \mathrm{~b}-\mathrm{d}$ & $19.36 \mathrm{bc}$ & \\
\hline \multirow[t]{3}{*}{200} & 0 & $16.36 f-i$ & $18.47 \mathrm{c}-\mathrm{g}$ & $19.19 c-f$ & $18.01 \mathrm{c}-\mathrm{e}$ & $20.37 a$ \\
\hline & 200 & $18.50 \mathrm{c}-\mathrm{g}$ & $20.75 b-e$ & $22.25 a-c$ & $20.50 \mathrm{~b}$ & \\
\hline & 300 & $19.08 c-f$ & $23.50 a b$ & $25.25 a$ & $22.61 \mathrm{a}$ & \\
\hline \multicolumn{2}{|c|}{$\mathrm{Zn}$} & $15.72 \mathrm{c}$ & $18.12 \mathrm{~b}$ & $20.38 a$ & & \\
\hline \multirow[t]{3}{*}{$A \times Z n$} & 0 & $14.11 \mathrm{f}$ & $16.16 \mathrm{de}$ & $18.60 \mathrm{bc}$ & & \\
\hline & 100 & $15.06 \mathrm{ef}$ & $17.30 \mathrm{~cd}$ & $20.32 a b$ & & \\
\hline & 200 & $17.98 \mathrm{~cd}$ & $20.91 a$ & $22.23 a$ & & \\
\hline \multirow[t]{3}{*}{$B \times Z n$} & 0 & $13.85 \mathrm{e}$ & 15.68de & $18.55 b c$ & & \\
\hline & 200 & $15.72 \mathrm{de}$ & $18.49 \mathrm{bc}$ & $20.24 b$ & & \\
\hline & 300 & $17.58 \mathrm{~cd}$ & $20.19 b$ & $22.36 a$ & & \\
\hline
\end{tabular}

Mean with a column, row and their interaction following with the same latter are not significantly different according to Duncan multiple at 0.05 level. 


\section{DISCUSSIONS}

The results of this research showed that, the most examined treatments had a positive influence on shoot lengths and diameters, leaf area, leaf dry weight, leaf chlorophyll content and number of leave per shoot. Increasing vegetative growth and leaf chlorophyll content by foliar spray with aminoplasmal may be attributed to the role of aminoplasmal as precursors and constituents of the proteins (Rai, 2002; El-Shabasi et al., 2005), which are important for stimulation of cell growth. They contain both acid and basic groups and act as buffers, which help to maintain favorable or indirectly influence the physiological activities in plant growth and development in increasing cell division and elongation and its role in enhancement of metabolite accumulation in leaves, also to increasing photosynthesis which leads to increase chlorophyll content in the leaves (Abd El-Aal et al., 2010). The present results are in agreement with the finding obtained by Molaie et al., (2013) who concluded that Amino acids are fundamental in chlorophyll production; Chlorophyll being the driving force behind photosynthesis. Amino acids help to increase chlorophyll concentration in the plant, leading to a higher degree of photosynthesis, which in turn leads to even more available energy and improve plant vegetative growth. In regard to Boron, it is observed from the results that Boron significantly increased shoot length, shoot diameter, Single leaf area, leaf dry weight, Total Leaf chlorophyll content and Number of leaves per shoot. The reasons behind this may be due to the role of boron has been long recognized as an essential element for plant growth, also boron has an effect on cell wall structure and has a major effect on cell elongation and transfer of sugar (Abdollahi et al., 2010). These results supported by findings by (Mazher et al., 2006; Roy et al., 2006 and Marschner, 1995) who showed that, boron improves necessary compounds for metabolic processes and building organs thereby vegetative growth. About Zinc results, these results were confirmed by (Maerschel et al., 2007) who found that application of Zinc had an important role in the formation of the auxin which produced by shoot tips, and controls cell division, leaf and shoot growth. In addition Zinc play as essential micronutrients required for optimum crop growth. It plays an important role in many biochemical reactions within the plants.

\section{REFERENCES}

- Abd El-Aal, F. S.; A. M. Shaheen; A.A. Ahmed and A.R. Mahmoud, (2010). Effect of foliar application of urea and amino acids mixtures as antioxidants on growth, yield and characteristics of squash. Research Journal of Agriculture and Biological Sciences. 6: 583-588.

- Abdollahi, M.; S. Eshghi and E. Tafazoli, (2010). Interaction of Paclobutrazol, Boron and Zinc on vegetative growth, yield and fruit quality of strawberry (Fragaria Ananassa Duch. Cv. Selva). Journal of Biodiversity and Environmental Sciences. 4(11): 67-75.

- Abo El-Magd, M.M. and E. Mona El-Azab, (2015). Comparison between foliage activator, root activator and soil fertilization in relation with onion growth, yield and quality of bulbs. Journal of Innovations in Pharmaceuticals and Biological Sciences. 2 (4): 411-425.

- Al-Rawi, A. A. and A. Khalaf-Alla, (2000). Analysis of Experimental Agriculture Design. Dar AlKutub for Printing and Publishing. Mosul University. Iraq.

- Brown, P.H.; L. Ferguson and G. Picchioni, (1995). Boron boosts pistachio yields. Fluid Journal. 4: 11-13.

- Coruzzi, G. and R. Last, (2000). Amino acids. In: Biochemistry and Molecular Biology of Plants. B. Buchanan, W. Gruissem, R. Jones (eds.). Amer. Soc. Plant Biol., Rockville, MD, USA (pub.). 358-410.

- El-Shabasi, M.S.; S.M. Mohamed and S.A. Mahfouz, (2005). Effect of Foliar Spray with Amino Acids on Growth, Yield and Chemical Composition of Garlic Plants. The 6th Arabian Conf. Hort., Ismailia,Egypt.

$-\mathrm{FAO}$

2015.

http://faostat.fao.org/site/567/default.aspx\#ancor

- Ganie, M.A. F. Akhter; M.A.Bhat; A.R. Malik; J.M. Junaid; M.A. Shah; A.H. Bhat and T.A. Bhat, (2013). Boron-a critical nutrient element for plant growth and productivity with reference to temperate fruits. Current Science 104(1): 76-85.

- Gobara, A.A. (1998). Response of Le-Cont Pear trees of foliar application of some nutrients. Egyptian journal of horticulture. 25: 55-70.

- Maerschel, R.; R. Kearsley Tomlinson and J. Wright, (2007). Zinc nutrition and plant growth. Available in website; www.spraygro.com.au.

- Marschner H (1986) Functions of mineral nutrients:acronutrients. International Research Journal 195-267. 
- Marschner, H. (1995). Mineral Nutrition.2nd ed. Academic Press. London, Great Britain. p. 889

- Mazher, A.A.M.; S.M Zaghloul,. and A.A. Yassen, (2006). Impact of boron fertilizer on growth and chemical constituents of Taxodiumdistichum grown under water regime.World Journal of Agriculture Science. 2 (4): 412- 420.

- Molaie, H.; B. Panahi and A .Tajabadipour, (2013). the effect of foliar application of some amino acid compounds on photosynthesis and yield of two commercial cultivars in pistachio orchards of Kerman province in Iran. International Journal of Agriculture and Crop Sciences. 5(23): 2827-2830.

- Neilsen, G.H.; D. Neilsen; E.J. Hogue and L.C. Herbert, (2004). Zinc and boron nutrition management in fertigated high density apple orchards. Canadian Journal of Plant Science. 84: 823-828.

- Rahdari, P. and B. Panahi, (2012). The evaluation of application amino acids on some quantitative and qualitative of pistachio. Journal of Iran Biology.25(4): 6006-6017.

- Rai, V.K. (2002). Role of amino acids in plant responses to stress. Biol.Plant, 45: 471-478.

- Ramos, D.E. 1997. Walnut production manual. Div. Agriculture Natural Sciences University of California, Oakland, CA.

- Roy,R.N.; A.Finck.; G.J. Blair and H.L.S Tandon, (2006). Plant nutrition for food security. Aguide for integrated nutrient management.Food and Agriculture Organizationof the United Nations, Rom.

- SAS, SAS/STAT (2001) "User's Guide for Personal Computer”.Release. 6.12. SAS Institute Inc, Cary, NC., U S A.

- Seyyedi, M. (1998). The effect of boron and zinc spraying on the yield and quality of pistachio fruit, Master thesis, Faculty of Agriculture, University of Tehran.

- Shiraishi, M.; H. Fujishima and H. Chijiwa, (2010). Evaluation of table grape genetic resources for sugar, organic acid,and amino acid composition of berries. Euphytica. 174: 1-13.

- Storey JB (2007) Zinc, In:A.V. Barker and D.J. Pilbeam (eds.). Handbook of plant nutrition. CRC Press,New York. p. 411-437.

- Wojcik, P. (2007). Vegetative and reproductive responses of apple trees to zinc fertilization under conditions of acid coarse-textured soil. Journal of Plant Nutrition. 30:1791-1802.

- Wojcik, P. and M. Wojcik, (2003). Effects of boron fertilization on Conference pear tree vigor, nutrition, and fruit yield and storability. Plant and Soil 256: 413-421.

- Zheng, W.; M.M. Pi and W.D. Liu. (1989). A study on the effects of boron on the carbon metabolism of Ramie.J. Huazhong Agr. Univ. 8:354-360. 